\title{
PIEZOELECTRIC EFFECTS IN GaN/AIGaN MULTIPLE QUANTUM WELLS PROBED BY PICOSECOND TIME-RESOLVED PHOTOLUMINESCENCE
}

\author{
H. S. Kim ${ }^{\text {a) }}$, J. Y. Lin $^{\dagger}$, H. X. Jiang ${ }^{\text {b) }}$, W. W. Chow*, A. Botchkarev**, and H. Morkoç** \\ ${ }^{\dagger}$ Department of Physics, Kansas State University, Manhattan, KS 66506-2601 \\ * Sandia National Laboratories, Albuquerque, NM 85718-0601 \\ ** Department of Electrical Engineering and Physics, Virginia Commonwealth University, \\ Richmond, Virginia 23284-3072
}

\section{Cite this article as: MRS Internet J. Nitride Semicond. Res. 4S1, G3.3 (1999)}

\begin{abstract}
Piezoelectric effects in GaN/AlGaN multiple quantum wells (MQWs) have been directly probed by picosecond time-resolved photoluminescence (PL) spectroscopy. The time-resolved PL spectra of the $40 \AA$ well MQWs reveal that the PL transition peak position is in fact blueshifted at early delay times due to the collective effects of quantum confinement of carriers, piezoelectric field, and Coulomb screening. However, the spectral peak position shifts toward lower energies as the delay time increases and becomes redshifted at longer delay times. By comparing experimental and calculation results, we have obtained a low limit of the piezoelectric field strength to be about $560 \mathrm{kV} / \mathrm{cm}$ in the $40 \AA$ well $\mathrm{GaN} / \mathrm{Al}_{0.15} \mathrm{Ga}_{0.85} \mathrm{~N}$ MQWs.
\end{abstract}

\section{INTRODUCTION}

The group III-nitride semiconductors have attracted much attention recently due to many important applications, such as blue/UV light emitting diodes (LEDs), laser diodes (LDs), and high-temperature/high-power electronic devices [1]. As demonstrated by GaN LDs, LEDs, and $\mathrm{GaN}$ based electronic devices, many GaN based devices must take advantage of multiple quantum well (MQW) structures such as GaN/AlGaN and InGaN/GaN MQWs. In order to optimize the device design, it is necessary to study and understand the physical properties of nitride MQWs as well as the MQW structural effects on the device performance. Recent work on the III-nitride alloy systems and MQWs has shown that localized excitons dominate the optical transitions in these systems at low temperatures[2-4]. And, it has been proposed and shown that piezoelectric fields due to lattice mismatch-induced strain in InGaN/GaN MQWs [5,6] and GaN/AlGaN QWs [6-9] are the primary reason for the large redshift of the photoluminescence (PL) emission peak. Indeed the piezoelectric field in strained layers has been used to modify the operating characteristics of devices fabricated from these materials [9$11]$.

\section{EXPERIMENT}

Time-resolved PL (TRPL) studies have been carried out for a set of GaN/AlGaN MQW samples grown under identical conditions with well thickness varying from 20 to $50 \AA$. These MQW samples were grown by reactive molecular beam epitaxy (MBE) on sapphire $\left(\mathrm{Al}_{2} \mathrm{O}_{3}\right)$ substrates with a $1.5 \mu \mathrm{m} \mathrm{Al}_{\mathrm{x}} \mathrm{Ga}_{1-\mathrm{x}} \mathrm{N}(\mathrm{x}=0.15)$ buffer layer. The MQWs are composed of ten periods of alternating $\mathrm{GaN}$ wells and $200 \AA \mathrm{Al}_{\mathrm{x}} \mathrm{Ga}_{1-\mathrm{x}} \mathrm{N}(\mathrm{x} \approx 0.15)$ barriers. All samples were nominally undoped and the GaN epilayers grown under similar conditions were semiinsulating. 
For PL measurements, samples were attached to copper sample holders and placed inside a closed-cycle He refrigerator with a temperature variation from 10 to $300 \mathrm{~K}$. Excitation pulses with pulse width of about $7 \mathrm{ps}$ at a repetition rate of $19 \mathrm{MHz}$ were provided by a cavitydumped dye laser (Coherent 702-2CD) with Rhodamine 6G dye solution, which was pumped by a yttrium-aluminum-garnet (YAG) laser (Coherent Antares 76) with a frequency doubler. The output from the dye laser was frequency doubled again to provide a tunable wavelength in the ultraviolet (UV) region. The laser output after the second doubler has an average power of about 30 $\mathrm{mW}$ and the laser beam size on the sample was about $50 \mu \mathrm{m}$ in diameter. The overall spectral resolution was about $0.2 \mathrm{meV}$ and the time resolution of the detection system was about 25 ps.

\section{RESULTS AND DISCUSSIONS}

\section{CW Spectra}

Low-temperature $(10 \mathrm{~K}) \mathrm{cw}$ PL spectra for four representative $\mathrm{GaN} / \mathrm{Al}_{\mathrm{x}} \mathrm{Ga}_{1-\mathrm{x}} \mathrm{N}$ MQW samples with well thickness $\mathrm{L}_{\mathrm{w}}=20$ $\AA$, $30 \AA, 40 \AA$, and $50 \AA$ are presented in Fig. 1(b), 1(c), 1(d), and 1(e), respectively. For comparison, the PL spectrum of a GaN epilayer grown under the similar conditions is also shown in Fig. 1(a). For the GaN epilayer, the dominant transition at 3.485 $\mathrm{eV}$ at $10 \mathrm{~K}$ is due to the recombination of the ground state of $\mathrm{A}$ exciton [12-15]. In the $20 \AA$ well MQW sample, the excitonic transition peak position at $10 \mathrm{~K}(3.548 \mathrm{eV})$ is blueshifted with respect to the epilayer by an amount of $63 \mathrm{meV}$, which is due to the well-known effects of quantum confinement of electrons and holes as well as the strain. Its transition energy (3.548 $\mathrm{eV}$ ) is comparable to the bandgap of $\mathrm{GaN}$ under $0.4 \%$ compressive strain, which is expected for $\mathrm{GaN}$ embedded between

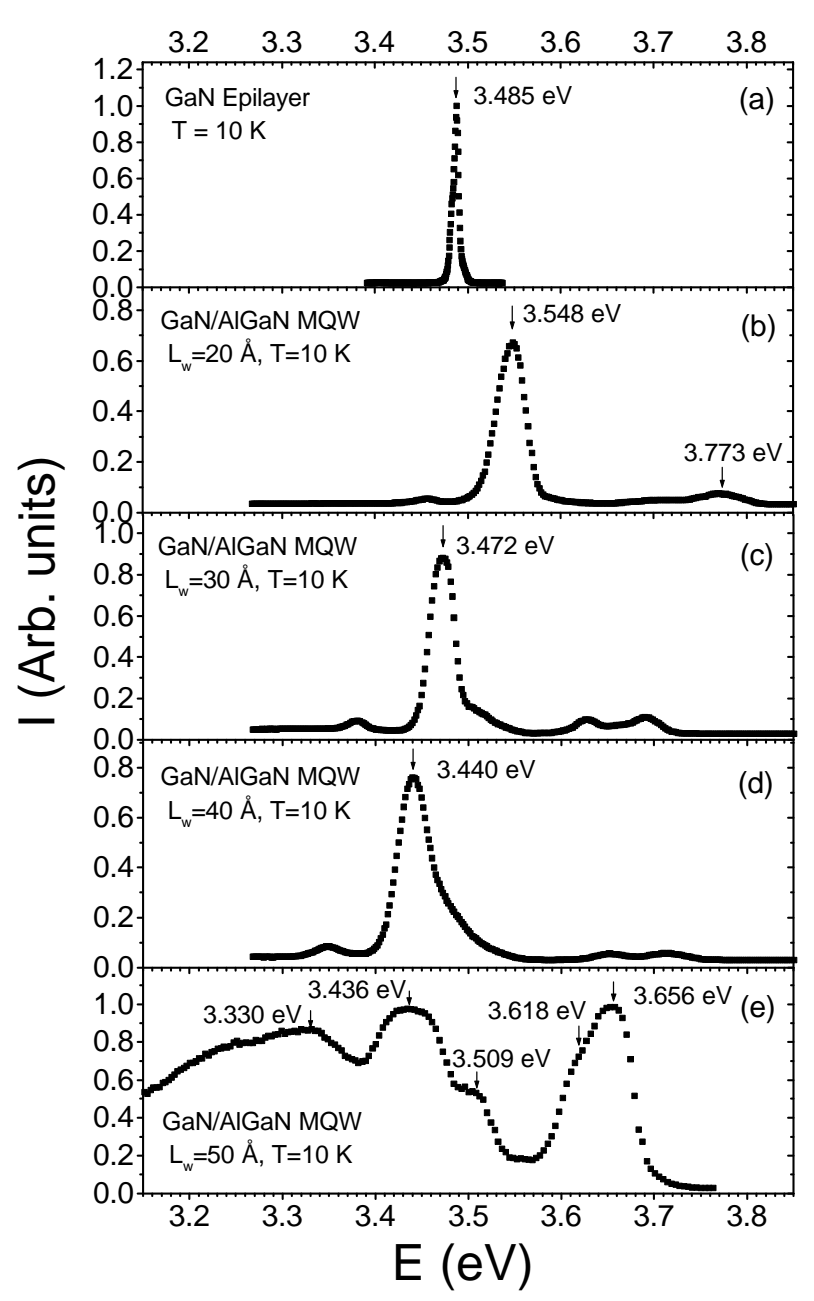

FIG. 1. Low-temperature $(10 \mathrm{~K}) \mathrm{cw}$ PL spectra of nominally undoped $\mathrm{GaN} / \mathrm{Al}_{\mathrm{x}} \mathrm{Ga}_{1-\mathrm{x}} \mathrm{N}$ MQW samples with well thickness (b) $\mathrm{L}_{\mathrm{w}}=20 \AA$, (c) $30 \AA$, (d) $40 \AA$, (e) $50 \AA$, and (a) GaN epilayer grown under identical conditions as the MQW samples taken under the same experimental conditions.

$\mathrm{Al}_{0.15} \mathrm{Ga}_{0.85} \mathrm{~N}$ layers [7]. In the $30 \AA$ and $40 \AA$ well MQW samples, the cw transition peak positions at $10 \mathrm{~K}$ are redshifted with respect to the $\mathrm{GaN}$ epilayer by an amount of 13 and 45 meV, respectively. The origin of this redshift will be discussed later in relation with the results of the time-resolved PL (TRPL) spectra. On the other hand, the PL spectrum of the $50 \AA$ well MQW sample exhibits a fairly complex behavior. The transition peak at higher emission energies (3.656 eV) in this sample is due to an optical transition in the AlGaN barrier regions [3]. The temperature dependence of the cw PL spectra of these samples has been measured. Figure 2 shows cw emission spectra of the $40 \AA$ well MQW samples measured at four 
representative temperatures $\mathrm{T}=10,100,200$, and $300 \mathrm{~K}$, respectively. We observed two emission lines $(3.440$ and $3.480 \mathrm{eV}$ at $\mathrm{T}=10 \mathrm{~K})$ with the lower-energy emission line being the dominant one at low temperatures. The higher-energy emission line may be related with higher

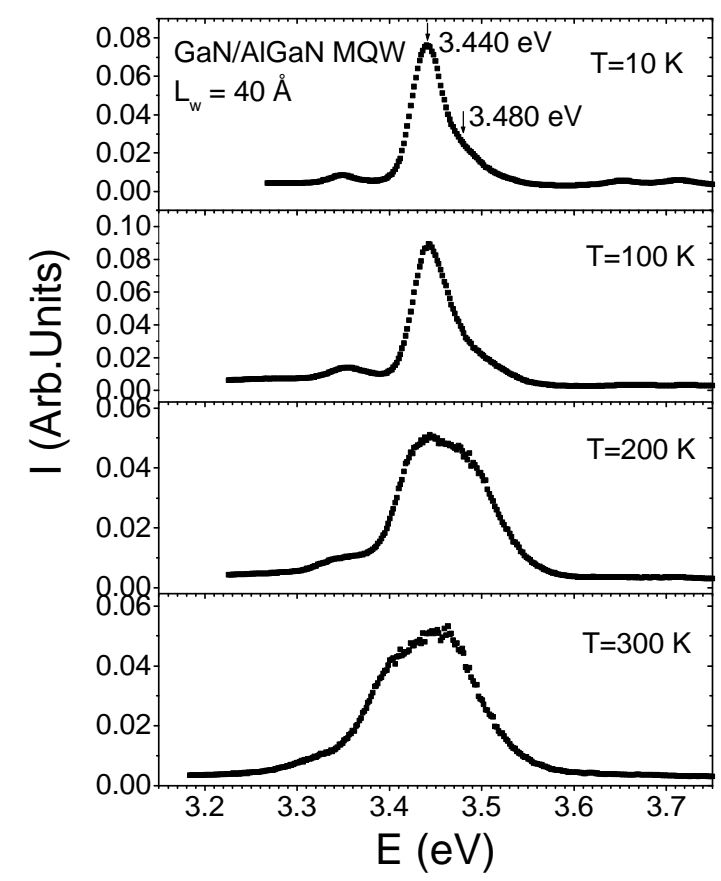

FIG. 2. CW PL spectra of nominally undoped 40 $\AA$ well $\mathrm{GaN} / \mathrm{Al}_{\mathrm{x}} \mathrm{Ga}_{1-\mathrm{x}} \mathrm{N}$ MQWs measured at four representative temperatures $\mathrm{T}=$ $10,100,200$, and $300 \mathrm{~K}$.

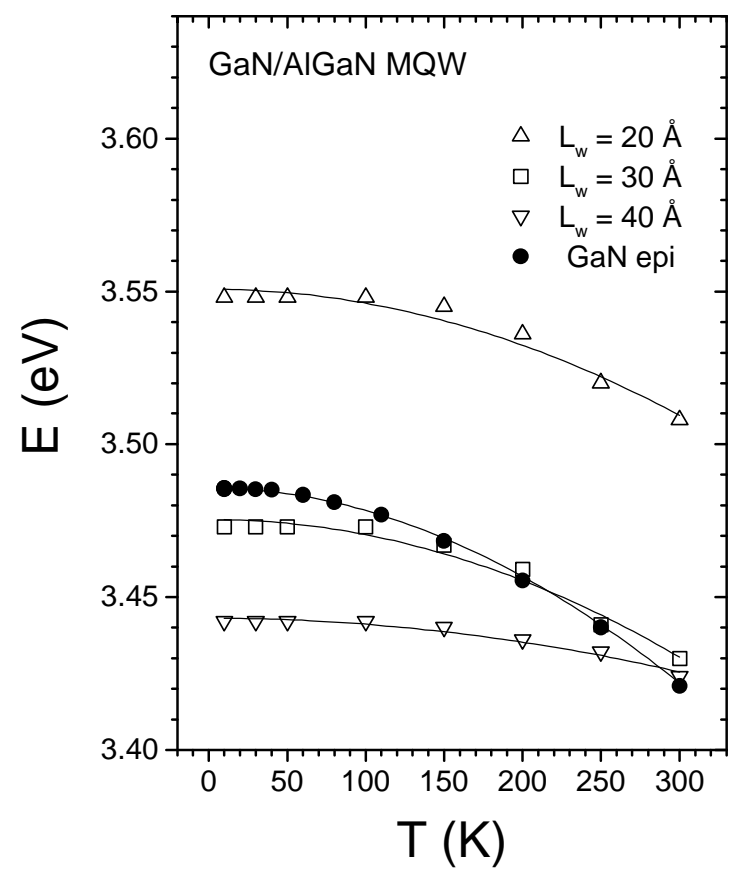

FIG. 3. The temperature dependence of the PL spectra peak positions of the dominant emission lines of $\mathrm{GaN} / \mathrm{Al}_{\mathrm{x}} \mathrm{Ga}_{1-\mathrm{x}} \mathrm{N} \mathrm{MQW}$ samples with well thickness $\mathrm{L}_{\mathrm{w}}=20 \AA, 30$ $\AA$, and $40 \AA$. The solid lines are the leastsquares fit of data with Eq. (1).

subband transitions and becomes dominant at higher temperatures $(\mathrm{T}=300 \mathrm{~K})$. The temperature dependence of the PL peak position of the dominant emission line in the $\mathrm{L}_{\mathrm{w}}=20 \AA, 30 \AA$, and $40 \AA$ MQWs has been plotted in Fig. 3. For comparison, the temperature dependence of the PL spectral peak of a GaN epilayer grown under the similar conditions is also shown in Fig. 3. The temperature dependence of the dominant emission peak positions can be fitted by the temperature dependence of the bandgap energy using the empirical equation [16],

$$
E_{0}(T)=E_{0}(0)-\alpha T^{2} /(\beta+T),
$$

where $E_{0}(0)$ is the transition energy at $T=0 \mathrm{~K}$ and $\alpha$ and $\beta$ are constants referred to as Varshni thermal coefficients. The solid curves in Fig. 3 are the least-squares fit of data by using Eq. (1). The fitted values for the GaN epilayer and MQWs with $\mathrm{L}_{\mathrm{w}}=20 \AA, 30 \AA$, and $40 \AA$ are $E_{0}(0)=$ $3.486 \mathrm{eV}, 3.551 \mathrm{eV}, 3.475 \mathrm{eV}$, and $3.443 \mathrm{eV} ; \alpha=0.0016 \mathrm{eV} / \mathrm{K}, 0.0010 \mathrm{eV} / \mathrm{K}, 0.0011 \mathrm{eV} / \mathrm{K}$, and $0.0011 \mathrm{eV} / \mathrm{K} ; \beta=1.89 \times 10^{3} \mathrm{~K}, 2.01 \times 10^{3} \mathrm{~K}, 2.01 \times 10^{3} \mathrm{~K}$, and $5.41 \times 10^{3} \mathrm{~K}$, respectively. The parameters obtained from the GaN epilayer and the $20 \AA$ and $30 \AA$ well MQWs are comparable to the parameters $\left(E_{0}(0)=3.486 \mathrm{eV}, \quad \alpha=8.32 \times 10^{-4} \mathrm{eV} / \mathrm{K}\right.$, and $\beta=835.6 \mathrm{~K}$ ) obtained by Shan et. al. [14] for a MOCVD GaN epilayer using the photoreflectance measurement. The value of $\beta$ for the $40 \AA$ well MQWs is anomalously larger 
than those for the GaN epilayer. It is clear that for the $20 \AA$ well MQW sample, the exciton peak position is blue shifted with respect to the epilayer at all temperatures. On the other hand, with respect to the GaN epilayer, the PL emission peaks in the $30 \AA$ and $40 \AA$ well MQW samples are redshifed with the amount of shift decreasing with temperature and become blue shifted at $300 \mathrm{~K}$. Such a behavior is related to the effect of piezoelectric fields in these MQWs to be discussed in more detail next.

\section{Time-resolved PL spectra}

Time-resolved emission spectroscopy was employed to study the dynamics of optical transitions in $\mathrm{GaN} / \mathrm{Al}_{\mathrm{x}} \mathrm{Ga}_{1-\mathrm{x}} \mathrm{N}$ MQWs. Figure 4 shows the TRPL spectra of the main emission lines of the $40 \AA$ well MQW samples measured at $T=10 \mathrm{~K}$ for several representative delay times. The arrows in Fig. 4 indicate the spectral peak positions at different delay times. The dotted line indicates the position of the excitonic transition peak in the GaN epilayer grown under similar conditions. Several features can be observed for the $40 \AA$ well MQW samples as

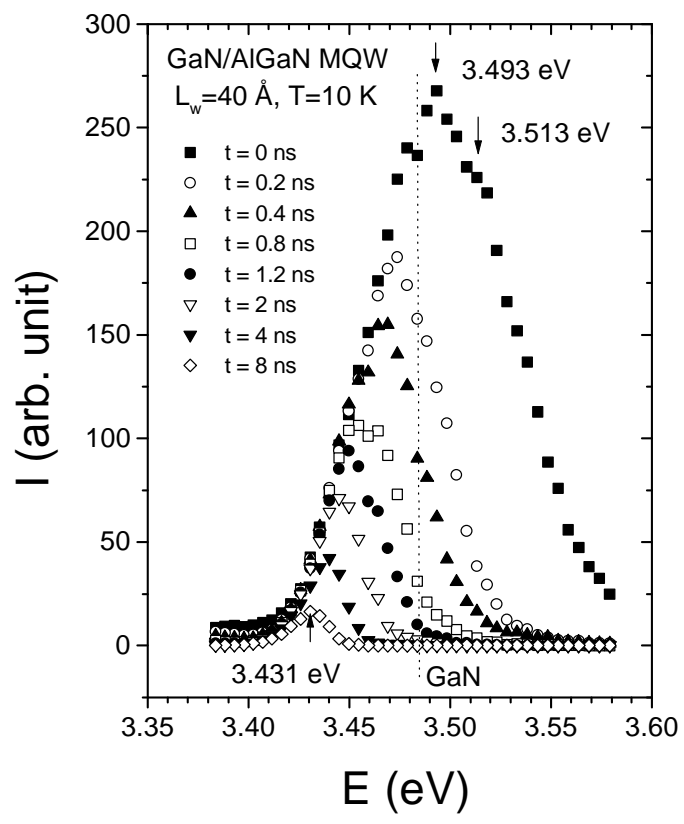

FIG. 4. Time-resolved PL spectra of the main emission lines measured at $T=10 \mathrm{~K}$ for $40 \AA$ well MQWs for several representative delay times. Here, the delay time $t_{d}=0$ has been chosen at the position of maximum intensity in the luminescence temporal responses. The arrows indicate the spectral peak positions at different delay times. The dotted lines indicate the position of the excitonic transition peak in GaN epilayers grown under similar conditions.

shown in Fig. 4. First, in contrast to the cw PL spectra shown in Fig.1(d), the spectral peak position at delay time $t_{d}=0(3.493 \mathrm{eV})$ is blueshifted with respect to the emission line in the $\mathrm{GaN}$ epilayer $(3.485 \mathrm{eV})$. Second, the linewith of the emission line decreases with delay time. Third, the peak position of the emission line markedly shifts toward lower energies with an increase of delay time. For comparison with different well thicknesses, we have plotted in Fig. 5 the delay time dependence of the peak position $E_{p}$ of different MQWs. In the case of $40 \AA$ and $50 \AA$ well MQW samples, $E_{p}$ shift toward lower energies for more than $60 \mathrm{meV}$ from $\mathrm{t}_{\mathrm{d}}=0$ to $t_{d}=7 \mathrm{~ns}$. The large amounts of redshift occurred in the early delay times, i.e., in the first $1 \mathrm{~ns}$ for $40 \AA$ MQWs and in the first 200 ps for $50 \AA$ MQWs.

The TRPL results can be explained well by the collective effects of piezoelectric field and photo-excited carrier screening. It is well established that piezoelectric fields are present in the well regions of the GaN/AlGaN MQWs and heterojunctions due to the lattice mismatch between $\mathrm{GaN}$ and $\mathrm{AlGaN}$ as well as the large value of the piezoelectric constant in GaN. Under 


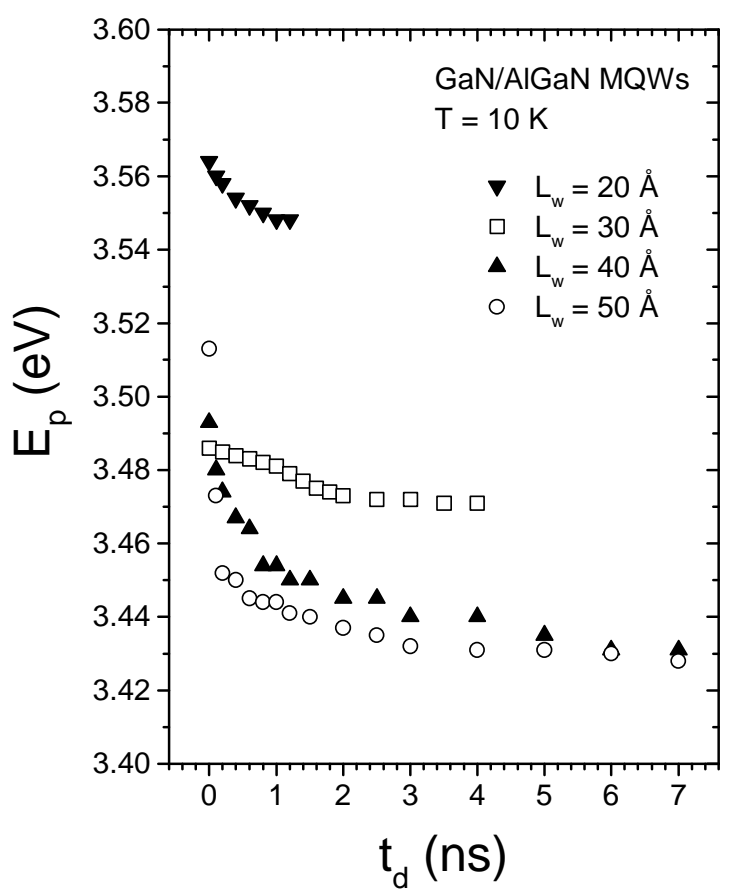

FIG. 5. The peak position $\mathrm{E}_{\mathrm{p}}$ of the main emission line as functions of delay time $t_{d}$ measured at $\mathrm{T}=10 \mathrm{~K}$ for the $20 \AA, 30 \AA, 40 \AA$, and $50 \AA$ well $\mathrm{GaN} / \mathrm{Al}_{\mathrm{x}} \mathrm{Ga}_{1-\mathrm{x}} \mathrm{N}$ MQW samples. the influence of the piezoelectric field, optically excited carriers drift apart. The electrons (holes) move toward the direction opposed to (along) the piezoelectric field and the field induced by these spatially separated charge carriers will screen the piezoelectric field. On the other hand, the screening field due to the spatially separated charge carriers decreases with delay time because of the radiative recombination of electrons and holes. At $t_{d}=0$, the screening field induced by the photoexcited electrons and holes is strongest, which reduces or partially balances out the piezoelectric field. As the delay time increases, carriers recombine radiatively and the screening field gradually diminishes and the original piezoelectric field restores. Thus the total amount of shift from $t_{d}=0$ to $t_{d} \rightarrow \infty$ effectively corresponds to the variation of the electron and hole energy levels in the presence of the piezoelectric field with and without carrier screening, respectively. From this the piezoelectric field strength can also be estimated. By comparing experimental and calculation results, a lower limit value of

the piezoelectric field strength of about $560 \mathrm{kV} / \mathrm{cm}$ in $\mathrm{GaN} / \mathrm{Al}_{0.15} \mathrm{Ga}_{0.85} \mathrm{~N}$ MQWs as well as the electron and hole wave functions have been obtained for a $40 \AA$ well MQW [8].

\section{CONCLUSIONS}

From cw PL spectra at low temperature $(10 \mathrm{~K})$, we observe that the exciton transitions for $20 \AA$ MQWs are blue shifted with respect to the GaN epilayer and the PL emission peak positions for $30 \AA, 40 \AA$, and $50 \AA$ well MQWs are redshifted with respect to the $\mathrm{GaN}$ epilayer. On the other hand, the time-resolved PL spectra of the $30 \AA, 40 \AA$, and $50 \AA$ well MQWs reveal that the excitonic transition is in fact blueshifted at early delay times due to quantum confinement of carriers and the spectral peak position shifts toward lower energies as the delay time increases and becomes redshifted at longer delay times. It has been demonstrated that the results described above are due to the presence of the piezoelectric field in the GaN wells of GaN/AlGaN MQWs subject to elastic strain together with screening of the photoexcited carriers and Coulomb interaction. Our results show that TRPL spectroscopy is a powerful tool for studying and understanding the piezoelectric effects on optical properties of GaN MQWs and heterostructures.

\section{ACKNOWLEDGMENTS}

The research at Kansas State University is supported by ARO, BMDO, DOE (96ER45604/A000), and NSF (DMR-95-28226 and INT-97-29582). The research at the Virginia Commonwealth University is supported by ONR and AFOSR. The research at Sandia National Laboratories is supported in parts by DOE under contract No. DE-AC04-94AL85000. 
H. S. Kim acknowledges support by RINS (BSRI-97-2406) in Gyeongsang National

University.

\section{REFERENCES}

a) On leave from Department of Physics, Gyeongsang National University, Chinju, Korea.

b) e-mail : Jiang@phys.ksu.edu

1. H. Morkoç, S. Strite, G. B. Gao, M.E. Lin, B. Sverdlov, and M. Burns, J. Appl. Phys. 76, 1363 (1994).

2. M. Smith, G. D. Chen, J. Y. Lin, H. X. Jiang, M. Asif Khan, and Q. Chen, Appl. Phys. Lett. 69, 2837 (1996).

3. M. Smith, J. Y. Lin, H. X. Jiang, A. Salvador, A. Botchkarev, W. Kim, and H. Morkoç, Appl. Phys. Lett. 69, 2453 (1996).

4. E. S. Jeon, V. Kozlov, Y. K. Song, A. Vertikov, M. Kuball, A. V. Nurmikko, H. Liu, C. Chen, R. S. Kern, C. P. Kuo, and M. G. Craford, Appl. Phys. Lett. 69, 4194 (1996).

5. T. Takeuchi, S. Sota, M. Katsuragawa, M. Komori, H. Takeuchi, H. Amano, and I. Akasaki, Jpn. J. Appl. Phys. Part 2 36, L382 (1997).

6. A. Hangleiter, J. S. Im, H. Kollmer, S. Heppel, J. Off, and F. Scholz, MRS Internet J. Nitride Semicond. Res. 3, 15 (1998).

7. Jin Seo Im, H. Kollmer, J. Off, A. Sohmer, F. Scholtz, and A. Hangleiter, Phys. Rev. B57, R9435 (1998).

8. H. S. Kim, J. Y. Lin, H. X. Jiang, W. W. Chow, A. Botchkarev, and H. Morkoç, Appl. Phys. Lett. 73, 3426 (1998).

9. E. T. Yu, G. T. Sullivan, P. M. Asbeck, C. D. Wang, D. Qiao, and S. S. Lau, Appl. Phys. Lett. 71, 2794 (1997).

10. R. Gaska, T. W. Yang, A. Osinsky, A. D. Bykhovski, and M. S. Shur, Appl. Phys. Lett. 71, 3673 (1997).

11. E. S. Hellman, MRS Internet J. Nitride Semicond. Res. 3, 11 (1998).

12. G. D. Chen, M. Smith, J. Y. Lin, H. X. Jiang, S. H. Wei, M. Asif Khan, and C. J. Sun, Appl. Phys. Lett. 68, 2784 (1996).

13. D. C. Reynolds, D. C. Look, W. Kim, A. Özgür, A. Botchkarev, A. Salvador, H. Morkoç, and D. N. Talwar, J. Appl. Phys. 80, 594 (1996).

14. W. Shan, T. J. Schmidt, X. H. Yang, S. J. Hwang, J. J. Song, and B. Goldenberg, Appl. Phys. Lett. 66, 985 (1995).

15. K. C. Zeng, J. Y. Lin, H. X. Jiang, A. Salvador, G. Popovici, H. Tang, W. Kim, and H. Morkoç, Appl. Phys. Lett. 71, 1368 (1997).

16. Y. P. Varshni, Physica 34, 149 (1967). 